[CONTRIBUtron from the Carbohydrate Laboratory, Bureau of Chemistry, UNiTed STates Department of Agriculture.]

\title{
THE PREPARATION OF XYLOSE FROM CORN COBS.
}

\author{
BY K. P. MONROE. \\ Received March 22, 1919
}

The radical improvement in the method of preparation of xylose from corn cobs which resulted from the work of LaForge and Hudson ${ }^{1}$ led to further experiments with the object in view to remove the adhesive gumand hence facilitate the crystallization of xylose obtained on subsequent hydrolysis-by some more convenient laboratory method than extraction in an autoclave at $160^{\circ} \mathrm{C}$. It has been found that this gum may be removed by digestion with dil. alkali at $100^{\circ}$, and that the residue yields on acid hydrolysis $8-10 \%$ crystalline xylose of a quality superior to that obtained by the previous methods. ${ }^{2}$ The sugar solution derived from hydrolysis of the residue is less colored than the similar solutions from the previous methods, and a moderate use of decolorizing char removes the color almost completely. Crystallization of the xylose also takes place with uniform readiness.

The method, which apparently has the advantage of uniform results, and combines the improvement introduced by LaForge and Hudson ${ }^{3}$ with the laboratory convenience of the previous method of Hudson and Harding, ${ }^{4}$ is as follows:

Two $\mathrm{kg}$. of broken but not necessarily ground cobs are digested with ro liters of $1 \%$ sodium hydroxide solution for $\mathrm{I} .5$ hours in a large copper vessel. The contents of the digestor are heated as rapidly as possible to boiling, and then simmered for the required length of time at approximately $100^{\circ}$. After draining off the dark brown extract ${ }^{5}$ the cobs are pressed as dry as possible and washed thoroughly with hot water. When 4 or 5 alternate pressings and washings have been carried out the cobs are practically free from alkaline extract.

This residue is hydrolyzed in the digester for two hours with 8-1o liters of boiling $4 \%$ (by volume) sulfuric acid. Approximately an equal volume of straw-colored solution is obtained after decanting through cheese cloth

${ }^{1}$ LaForge and Hudson, J. Ind. Eng. Chem., 10, 925 (1918).

2 Fudson and Harding, Thrs Jorrnal, 40, i60I (I9I8); LaForge and Hudson, Loc. cit. See also Stone and Lotz, Am. Chem. J., I3, 348 (I89r). The somewhat anomalous fact that corn cob residue after removal of some of the xylan by neutral or alkaline extraction furnishes a more convenient source for crystalline xylose than the original material scrves to call attention to our ignorance of the exact nature of the various polysaccharides in the plant tissues, and the mechanism of their hydrolysis.

${ }^{3}$ LaForge and Fudson, Loc. cit.

4 Hudson and Harding, Ibid.

$525-30 \%$ of the material in the cobs is removed by this digestion. The sodiumgum compound contained in the extract is being further investigated. 
pressing and washing. This liquid is simmered in a copper kettle with an excess of barium carbonate until neutral to Congo red paper. ${ }^{1}$ About two hours are required for this operation; the heating serving the dual purpose of accelerating the reaction, which is necessarily slow on account of the slight solubility of barium carbonate, and digesting the barium sulfate so that it may be filtered and washed readily on large Büchner funnels provided with asbestos mats on filter paper. Occasional stirring is desirable during this digestion.

The pale yellow filtrate and washings are acidulated with a few drops of phosphoric acid, heated nearly to boiling and decolorized with bone black or preferably "Norit." 5 o to Ioo g. of "Norit" is required for the large volume of liquid. A mere trace of color remains in the filtrate from the decolorizing char, which is evaporated in vacuo to a pale yellow sirup of approximately $80 \%$ solids. $^{3,4}$ Crystallization of xylose occurs almost at once on seeding and stirring after mixing with approximately $1 / 2$ volume of glacial acetic acid. The crystalline xylose, filtered after standing overnight, is pure white when washed with a moderate amount of glacial acetic acid and, finally, absolute alcohol. 8 to I0\% crystalline xylose is so obtained.

Although the quality of this product is sufficient for most uses, the xylose may be conveniently purified by recrystallization. The crystals are dissolved in warm distilled water, the solution is poured into 4 volumes of $95 \%$ alcohol and the mixture filtered from traces of mineral matter. On evaporation in vacuo to an $80 \%$ sirup, stirring in $95 \%$ alcohol and seeding, crystallization takes place at once. After filtration, washing with $95 \%$ and absolute alcohol, the crystals are dried in a vacuum oven for 3 to 4 hours at a temperature not exceeding $50^{\circ}$.

WASHINGTON, D. C.

I The procedure of Hudson and Harding (Loc. cit.) to neutralize with lime at this point is an alternative method. Use of barium carbonate has the advantage that the solution can never become alkaline-in which case the preparation is usually a casualty -and barium sulfate may be quantitatively removed by filtration, dispensing with the procedure of pouring into alcohol.

2 When a copper vessel is used for the acid hydrolysis the solution is saturated with hydrogen sulfide and the cupric sulfide removed by filtration before decolorizing.

${ }^{3}$ Faint turbidity in the sirup is disregarded since it has no deleterious effect on the crystallization of xylose. If the mineral matter separating during the evaporation is appreciable, as is the case when calcium salts are present in the barium carbonate, the solution is poured into 4 volumes of $95 \%$ alcohol when a volume of approximately one litre is reached, and the inorganic salts are separated by filtration.

${ }^{4}$ It is of interest to note that the optical rotation of this solution is much higher than may be accounted for by the xylose, which suggests that some glucose may be produced from corn cob cellulose during the hydrolysis. 